\title{
Prevalence of anti-histone antibodies, their clinical significance and correlation with other autoantibodies in a cohort of Italian scleroderma patients
}

\author{
Gabriella Morozzi • Francesca Bellisai - Irene Fineschi - Francesca Scaccia • \\ Gabriella Pucci · Antonella Simpatico • Marilina Tampoia • Alessandra Chialà • \\ Giovanni Lapadula $\cdot$ Mauro Galeazzi
}

Received: 24 August 2010/Accepted: 13 January 2011/Published online: 23 March 2011

(C) Springer-Verlag 2011

\begin{abstract}
Purpose The aim of our study was to determine the prevalence, clinical significance of antibodies to individual histone components and to evaluate their correlation with other autoantibody specificities in a cohort of Italian SSc patients. Some authors, demonstrated high prevalence of anti-histone antibodies in Italian SSc patients, associated with cardiac and renal involvement, suggesting a prognostic value of these autoantibodies; however, these data need to be confirmed.

Methods Serum from 112 adult SSc patients, classified as diffuse (dc) and limited cutaneous (lc) SSc subsets were analyzed for autoantibodies by indirect immunofluorescence, fluoroenzyme immunoassay and enzyme immunoassay.

Results AHA were found in 13 patients (11.6\%), nine with $\mathrm{lcSSc}$ and four with dcSSc. Among them, five patients were anti-Scl70+ and four were anti-CENP B+. The presence of AHA was not associated with multi-organ involvement or with diffuse subset, as already described. Anti-Scl70 was detected in $43 \%$ of patients, anti-CENP B in $32 \%$ and anti-RNA polymerase III in $7.1 \%$. We
\end{abstract}

G. Morozzi ( $₫)$ · F. Bellisai · I. Fineschi · F. Scaccia •

G. Pucci - A. Simpatico - M. Galeazzi

Sezione di Reumatologia, Dipartimento di Medicina

Clinica e Scienze Immunologiche, Policlinico Le Scotte,

Viale Bracci, 53100 Siena, Italy

e-mail:morozzi@unisi.it

M. Tampoia · A. Chialà

Patologia Clinica I, Ospedale Policlinico-Bari, Bari, Italy

G. Lapadula

Sezione di Reumatologia, Dipartimento di Medicina

Interna e Medicina Pubblica (DiMIMP), Bari, Italy confirmed the association between anti-Scl70 antibodies and pulmonary fibrosis (OR 15.75, $p<0.0001$ ).

Conclusion In our experience, the very low prevalence of AHA in Italian SSc patients and the lack of association with clinical manifestations suggest that this test is of little clinical use; however, it would be worthwhile extending the study to a larger population of patients.

Keywords Systemic sclerosis - Anti-histone antibodies . Prevalence $\cdot$ Clinical manifestations

\section{Introduction}

The highest prevalence of anti-histone antibodies (AHA) is in drug-induced systemic lupus erythematosus (SLE), however, AHA are found also in other diseases, such as systemic sclerosis $(\mathrm{SSc})$. SSc is a systemic connective tissue disease characterized by microvascular injury and abnormalities of the immune system leading to fibrosis of the skin and major internal organs. Antinuclear autoantibody (ANA) specificities are associated with certain clinical features of the disease. Moreover, differences in the frequencies of ANA specificities correlated with ethnic origin and geographic locations are characteristic of the disease [1-6]. Some authors [6] have shown differences in antibody specificities and their clinical manifestation, between French and American Caucasian patients; others [7] have shown that serologic profiles of Swedish SSc patients did not differ significantly from those of Caucasian populations from other parts of the world. Large geographical variations in SSc prevalence and incidence have been described and differences in individual disease presentations were recently studied [8-10]. The latter analysis suggested that eastern European centers see SSc patients 
with more severe manifestations than other centers focusing on the major autoantibodies [10]. In diffuse SSc, positivity for IgG antibodies against inner core molecules of histone, such as H2B, is linked to severe lung fibrosis [7, 11, 12]. Parodi et al. [13] 15 years ago demonstrated $41.8 \%$ of AHA prevalence in Italian SSc patients, associated with cardiac and renal involvements, suggesting a prognostic value for these autoantibodies; the same group in 2002 [14] demonstrated that AHA alone or associated with anticentromere antibodies characterized a disease subset with more frequent visceral involvement and a probably poor outcome. The aim of our study was to determine the prevalence and clinical significance of antibodies to individual histone components and their correlation with other autoantibody specificities, in a cohort of Italian SSc patients.

\section{Materials and methods}

Our study included 112 adult Caucasian patients with SSc consecutively diagnosed in two Italian Rheumatology Units (Siena and Bari) in 2007 and 2008. All patients (99 females, 13 males) fulfilled the American College of Rheumatology criteria and were clinically grouped according to the classification of Leroy and Medsger [15, 16]. Mean age at the time of diagnosis was $56.3 \pm 12.2$ years and mean duration of disease was $8.1 \pm 4.7$ years. Serum samples were analyzed by routine tests and stored at $-40^{\circ} \mathrm{C}$ until serological assay. All patients gave written informed consent for anonymous storage of serum. Clinical data were obtained by retrospective review of the records; the study was performed according to guidelines issued by local ethical committee. Onset of the disease was regarded as the time of the first non-Raynaud symptom. Esophageal motility was assessed by double-contrast esophagography and manometric measurements. Lung fibrosis was studied by conventional radiography and functional tests. Pulmonary hypertension was defined according to the ESC guidelines of Venice, 2003 [17]. Electrocardiography and echocardiography were used to investigate pulmonary hypertension. The echocardiographic cut-off was $35 \mathrm{mmHg}$ at rest, which is higher than $25 \mathrm{mmHg}$ considered in the definition. Right heart catheterisation was performed only in those patients with values higher than $40 \mathrm{mmHg}$ to identify other cardiac problems and to prescribe treatment with ETRA. Renal function was determined by routine biological tests and by echography. Radiographs of hands, feet, elbows and knees were used to investigate joint involvement and calcinosis. Muscular involvement was evaluated by CPK and specific electromyographic alterations. Multi-organ involvement was recorded when more than one organ was involved without considering Raynaud phenomenon.
Serological tests

The sera were tested by indirect immunofluorescence for ANA at a serum dilution of 1:160, using human HEp-2 cell line as substrate (INOVA, San Diego, USA) and by Western immunoblot (HEp-2 Marblot strips, MarDx, CA, USA). In particular, the last technique allows detection also of anti-Ku and anti-fibrillarin specificities. Anti-ENA and anti-Scl70 was detected by fluoro-enzyme immunoassay (FEIA) (PHADIA, Germany). Anti-PM/Scl, anti-RNA polymerase III and anti-histone components were tested by ELISA (AESKU Diagnostics, Germany; MBL, Japan; and AESKU, respectively). Anti-histone components (H1, $\mathrm{H} 2 \mathrm{~A}, \mathrm{H} 2 \mathrm{~B}, \mathrm{H} 3$ and $\mathrm{H} 4$ ) were derived from human native protein extracted from HeLa cell lines, as declared by AESKU.

\section{Statistical analysis}

Chi-square test or Fisher's exact test were used to determine significant differences between sets of categorical data. The odds ratio was calculated using Woolf's method to establish associations between autoantibodies and organ involvement.

\section{Results}

Out of 112 patients, $47(42 \%)$ had diffuse cutaneous SSc (dcSSc) and $65(58 \%)$ had limited cutaneous SSc (lcSsc). Frequent clinical features were lung fibrosis (65\%), gastrointestinal involvement $(63 \%)$, pulmonary hypertension (39\%), and multi-organ involvement (52\%) (Table 1). None of our patients was diagnosed with SSc and other connective tissue diseases. Four patients died. Two had diffuse disease (anti-Scl70+, one died of kidney failure, one of severe lung fibrosis), while the other two had limited disease (anti-Scl70+, one patient died of lung neoplasm and one of pulmonary hypertension). A total of 109 (97.4\%) patients were ANA positive. The most common ANA patterns were "diffuse grains" (42\%), centromeric (32\%), speckled $(8.9 \%)$, nucleolar $(9.8 \%)$, and homogeneous (4.5\%); we also found cytoplasmic pattern (3.6\%) and mitotic spindle apparatus pattern (1.7\%) (Table 2). All anticentromere positive sera by IIF were confirmed by FEIA as anti-CENP B positive. AHA were found in 13 patients (11.6\%), 9 with limited disease and 4 with diffuse disease; 5 were also anti-Scl70+ and 4 were anti-CENP B+. AHA antibodies were not significantly associated with lung fibrosis or related to multi-organ involvement or any other autoantibody specificity. In the 13 AHA-positive patients, antibody specificities were: nine $\mathrm{H} 1$ (2 dcSSc, $7 \mathrm{lcSSc}$ ), seven $\mathrm{H} 2 \mathrm{~B}$ ( $3 \mathrm{dcSSc}, 4 \mathrm{lcSSc}$ ), one H2A (lcSSc), two H3 
Table 1 Clinical features frequent in 112 Italian patients

${ }^{a}$ Hypertension and/or raised serum creatinine level

Table 2 Distribution of IIF patterns and specificities of autoantibodies in 112 Italian patients

\begin{tabular}{lclll}
\hline Clinical manifestations & N/total (\%) & $\begin{array}{l}\text { Diffuse } 47 \\
(41.9) ~ N / \text { diff }(\%)\end{array}$ & $\begin{array}{l}\text { Limited 65 } \\
(58) N / \text { lim (\%) }\end{array}$ & $p$ \\
\hline Gastrointestinal involvement (esophagus) & $71(63)$ & $29(62)$ & $42(65)$ & $\mathrm{ns}$ \\
Lung fibrosis & $73(65)$ & $40(85)$ & $33(50.7)$ & $<0.01$ \\
Pulmonary hypertension & $44(39)$ & $23(49)$ & $21(32)$ & $0.08(\mathrm{~ns})$ \\
Renal involvement $^{\mathrm{a}}$ & $4(3.6)$ & $4(8.5)$ & - & - \\
Myositis $_{\text {Multi-organ involvement }}$ & $4(3.6)$ & $4(8.5)$ & - & - \\
Renal crisis & $59(52)$ & $31(65.9)$ & $28(43)$ & $<0.01$ \\
Deceased & $2(1.7)$ & $2(4.2)$ & - & - \\
\hline
\end{tabular}

\begin{tabular}{lccll}
\hline $\begin{array}{l}\text { IIF patterns and specificities } \\
\text { of autoantibodies }\end{array}$ & N/total $(\%)$ & $\begin{array}{l}\text { Diffuse } \\
\text { N/total }(\%)\end{array}$ & $\begin{array}{l}\text { Limited } \\
\text { N/total (\%) }\end{array}$ & $p$ \\
\hline Positive IIF ANA & $109(97)$ & $45(96)$ & $64(98)$ & $\mathrm{ns}$ \\
Diffuse grains & $47(42)$ & $23(49)$ & $24(37)$ & $\mathrm{ns}$ \\
ACA & $36(32)$ & $8(17)$ & $28(43)$ & $<0.01$ \\
Speckled & $10(8.9)$ & $6(13)$ & $4(6)$ & $\mathrm{ns}$ \\
Nucleolar & $11(9.8)$ & $5(11)$ & $6(9)$ & $\mathrm{ns}$ \\
Homogeneous & $5(4.5)$ & $3(6)$ & $2(3)$ & $\mathrm{ns}$ \\
Mitotic spindle apparatus & $2(1.7)$ & $1(2)$ & $1(1.5)$ & - \\
Cytoplasmic & $4(3.6)$ & $1(2)$ & $3(5)$ & - \\
More than one positive IIF pattern & $11(9.8)$ & $3(6)$ & $8(12)$ & $\mathrm{ns}$ \\
Specificities identified & & & & $\mathrm{ns}$ \\
Anti-SCl70 & $48(43)$ & $24(51)$ & $24(37)$ & $<0.01$ \\
Anti-CENPB & $36(32)$ & $8(17)$ & $28(43)$ & $\mathrm{ns}$ \\
Anti-histones & $13(11.6)$ & $4(8.5)$ & $9(14)$ & $\mathrm{ns}$ \\
Anti-RNA pol III & $8(7.1)$ & $4(8.5)$ & $4(6)$ & - \\
Anti-PM/SCL & $4(3.5)$ & $3(6.3)$ & $1(1.5)$ & - \\
Anti-fibrillarin & $2(1.8)$ & $1(2)$ & $1(1.5)$ & - \\
Anti-Ku & $1(0.9)$ & $1(2.1)$ & - & - \\
\hline
\end{tabular}

(lcSSc) and one H4 (lcSSc). Anti-Scl70 and nucleolar pattern were associated with multi-organ involvement $(\mathrm{OR}=3.92, p<0.01$ and $\mathrm{OR}=4.79, p<0.01$, respectively). Anti-Scl70 antibodies were associated with lung fibrosis $(\mathrm{OR}=15.75, p<0.0001)$. There was a difference in organ involvement and ANA specificities between patients with dcSSc and $\mathrm{lcSSc}(p<0.01)$ and of $17 / 28$ lcSSc patients with multi-organ involvement were antiScl70 positive $(61 \%, \mathrm{OR}=5.6, p<0.01)$.

\section{Discussion}

In this study of Italian SSc patients, anti-histone autoantibodies did not show a remarkable prevalence. These antibodies were not statistically associated with lung fibrosis, multi-organ involvement or any other autoantibody specificity, conflicting with results obtained by other authors [7, 11, 13, 14]. In fact, in the study by Parodi et al. on the prevalence of AHA in SSc patients, AHA was associated with ACA and anti-Scl70 more frequently than with other ANA, and Sato et al. reported AHA in 27 of 92 patients with SSc. Our results did not support these previous results suggesting that it is unproductive to look for AHA in scleroderma, probably because these antibodies are only an epiphenomenon of the disease. Moreover, we need to consider that since the 1950s when autoantibodies that bind to histones have been demonstrated, a variety of assays have been used to detect AHA. Initially, immunofixation or complement fixation methods were used; more recently, RIA, immunoblotting, and EIA methods have been developed. Different analytic methods have yielded discrepant results. Thus, outside a research setting, there are insufficient data to support the use of testing for AHA for the diagnostic or prognostic purposes. 
Concerning other autoantibodies, the frequency of ANA in our cohort of patients was $97.4 \%$, in line with previous studies [6]. Anti-Scl70 was positive in $43 \%$ of patients with SSc. This frequency was approximately equal to that found in the EUSTAR study [10] for the Italian subgroup (from Milan) and to previous studies on French Caucasian patients, and was higher than in US Caucasian patients [6]. Conversely, our patients with SSc had a relatively higher percentage of ACA (32\%) than patients of other studies (16-22\%) [6, 18]. In particular, statistically significant differences in the distribution of major antibodies (antiScl70, anti-centromere and anti-RNA pol III) were observed between French and American Caucasian patients. In the latter population, the three autoantibodies had quite similar distribution, whereas in the French population, as well in the present study population, anti-Scl70 was more frequent than anti-centromere, and anti-RNA pol III was less prevalent [6]. We also found that "diffuse grains" was the typical fluorescence pattern of anti-Scl70 antibodies, not the homogeneous/nucleolar pattern described by other authors. This may explain the different prevalence of nucleolar pattern found in our study; the association with multi-organ involvement, however, was in line with other studies [7]. The prevalence of anti-fibrillarin antibodies was low and comparable with that reported in other studies [6, 18].

Anti-Scl70 and anti-RNA pol III are representative SSc-specific antibodies linked to dSSc. Both are associated with diffuse skin thickening, but vasculopathy and pulmonary fibrosis are more frequently seen in patients with anti-Scl70 [18]. Results of the present study confirm these findings. In the literature, the rate of renal crisis has been significantly associated with anti-RNA pol [18], whereas in our sample we observed two renal crises, both in patients with anti-Scl70 antibodies, as previously showed in other study in Italy [19]. When we compared clinical features with SSc-related ANAs, in line with previous reports, we found strong associations between SSc-related ANA and clinical features: anti-Scl70 with $\mathrm{dSSc}$, and lung fibrosis and multi-organ involvement with dcSSc and lcSSc. In our cohort of patients, pulmonary hypertension was often secondary to lung fibrosis, and we therefore did not find correlations with anti-centromere antibodies.

In conclusion, our data indicate that: AHA have very low prevalence in Italian SSc patients and it is therefore unproductive to look for them in this disease; due to low prevalence, anti-fibrillarin and anti-PM-Scl autoantibody tests should be performed only in the presence of clinical symptoms strongly suggestive of SSc and/or in reference centers. Nowadays the lack of standardization of methods (Western blot/immunodot, etc.) could increase the risk of false positive and clinical misinterpretation. More studies with larger samples could be useful to standardize the measurement of these autoantibodies, basically because their detection can be helpful for diagnosing patients who test negative for anti-Scl70 and anti-centromere antibodies.

Conflict of interest None.

\section{References}

1. Bunn CC, Denton CP, Shi-Wen X, Knight C, Black CM (1998) Anti-RNA polymerases and other autoantibody specificities in systemic sclerosis. Br J Rheumatol 37:15-20

2. Jacobsen S, Halberg P, Ullman S et al (1998) Clinical features and serum antinuclear antibodies in 230 Danish patients with systemic sclerosis. Br J Rheumatol 37:39-45

3. Tager RE, Tikly M (1999) Clinical and laboratory manifestations of systemic sclerosis (scleroderma) in black South Africans. Rheumatology 38:397-400

4. Kuwana M, Okano Y, Kaburaki J, Tojo T, Medsger TA Jr (1994) Racial differences in the distribution of systemic sclerosis-related serum antinuclear antibodies. Arthritis Rheum 37:902-906

5. Kuwana M, Kaburaki J, Arnett FC, Howard RF, Medsger TA Jr, Wright TM (1999) Influence of ethnic background on clinical and serologic features in patients with systemic sclerosis and antiDNA-topoisomerase I antibody. Arthritis Rheum 42:465-474

6. Meyer OC, Fertig N, Lucas M, Somogyi N, Medsger TA Jr (2007) Disease subsets, antinuclear antibody profile, and clinical features in 127 French and 247 US adult patients with systemic sclerosis. J Rheumatol 34:104-109

7. Hesselstrand R, Scheja A, Shen GQ, Wiik A, Akesson A (2003) The association of antinuclear antibodies with organ involvement and survival in systemic sclerosis. Rheumatology (Oxford) 42:534-540

8. Chifflot H, Fautrel B, Sordet C, Chatelus E, Sibilia J (2008) Incidence and prevalence of systemic sclerosis: a systematic literature review. Semin Arthritis Rheum 37:223-235

9. Arias-Nuñez MC, Llorca J, Vazquez-Rodriguez TR et al (2008) Systemic sclerosis in northwestern Spain: a 19-year epidemiologic study. Medicine (Baltimore) 87:272-280

10. Walker UA, Tyndall A, Czirják L et al, EUSTAR co-authors (2009) Geographical variation of disease manifestations in systemic sclerosis: a report from the EULAR Scleroderma Trials and Research (EUSTAR) group database. Ann Rheum Dis 68:856-862

11. Sato S, Ihn H, Kikuchi K, Kikuchi K, Takehara K (1994) Antihistone antibodies in systemic sclerosis: association with pulmonary fibrosis. Arthritis Rheum 37:391-394

12. Hasegawa M, Sato S, Kikuchi K et al (1998) Antigen specificity of antihistone antibodies in systemic sclerosis. Ann Rheum Dis 57:470-475

13. Parodi A, Drosera M, Barbieri L, Rebora A (1995) Antihistone antibodies in scleroderma. Dermatology 191:16-18

14. Parodi A, Drosera M, Barbieri L, Rebora A (2002) Scleroderma subsets are best detected by the simultaneous analysis of the autoantibody profile using commercial ELISA. Dermatology 204(1):29-32

15. Anonymous (1980) Preliminary criteria for the classification of systemic sclerosis (scleroderma). Subcommittee for scleroderma criteria of the American Rheumatism Association Diagnostic and Therapeutic Criteria Committee. Arthritis Rheum 23:581-590

16. LeRoy EC, Black C, Fleischmajer R et al (1988) Scleroderma (systemic sclerosis): classification, subsets and pathogenesis. J Rheumatol 15:202-205 
17. Simonneau G, Robbins IM, Beghetti M et al (2009) Updated Clinical Classification of Pulmonary Hypertension. JACC 54 (1), Suppl S:S43-S54

18. Steen VD (2005) Autoantibodies in systemic sclerosis. Semin Arthritis Rheum 35:35-42
19. Codullo V, Cavazzana I, Bonino C et al (2009) Serologic profile and mortality rates of scleroderma renal crisis in Italy. J Rheumatol 36:1464-1469 